\title{
Spectral asymmetry for bag boundary conditions
}

\author{
C G Beneventano ${ }^{1}$, E M Santangelo ${ }^{1}$ and A Wipf ${ }^{2}$ \\ ${ }^{1}$ Departamento de Física, Universidad Nacional de La Plata, CC67, 1900 La Plata, Argentina \\ 2 Theoretisch-Physikalisches Institut, Friedrich-Schiller-Universität Jena, D-07743 Jena, \\ Germany \\ E-mail: gabriela@obelix.fisica.unlp.edu.ar, mariel@obelix.fisica.unlp.edu.ar \\ andwipf@tpi.uni-jena.de
}

Received 29 May 2002

Published 22 October 2002

Online at stacks.iop.org/JPhysA/35/9343

\begin{abstract}
We give an expression, in terms of boundary spectral functions, for the spectral asymmetry of the Euclidean Dirac operator in two dimensions, when its domain is determined by local boundary conditions and the manifold is of product type. As an application, we explicitly evaluate the asymmetry in the case of a finitelength cylinder and check that the outcome is consistent with our general result. Finally, we study the asymmetry in a disc, which is a non-product case, and propose an interpretation.
\end{abstract}

PACS numbers: $02.30 . \mathrm{Tb}, 02.30 . \mathrm{Sa}$

Mathematics Subject Classification: 35P05, 35J55

\section{Introduction}

Spectral functions are of interest both in quantum field theory and in mathematics (for a recent review, see [1]). In particular, $\zeta$-functions of elliptic boundary problems are known to provide an elegant regularization method [2] for the evaluation of objects as one-loop effective actions and Casimir energies, as discussed, for instance, in the reviews [3].

In the case of operators with a non-positive-definite principal symbol, another spectral function has been studied, known as the $\eta$-function [4], which characterizes the spectral asymmetry of the operator. This spectral function was originally introduced in [5], where an index theorem for manifolds with boundary was derived. In fact, the $\eta$-function of the Dirac operator, suitably restricted to the boundary, is proportional to the difference between the anomaly and the index of the Dirac operator, acting on functions satisfying non-local AtiyahPatodi-Singer (APS) boundary conditions. Some examples of application were discussed in $[6,7]$.

Such non-local boundary conditions were introduced mainly for mathematical reasons, although several applications of this type of boundary value problems to physical systems have emerged, ranging from one-loop quantum cosmology [8], fermions propagating in 
external magnetic fields [9] or so-called $S$-branes, which are mapped into themselves under $T$-duality [10]. So far, $\eta$-functions have found their most interesting physical applications in the discussion of fermion number fractionization [11]: the fractional part of the vacuum charge is proportional to $\eta(0)$. The $\eta$-function also appears as a contribution to the phase of the fermionic determinants and, thus, to effective actions [12]. Furthermore, both the index and the $\eta$-invariant of the Dirac operator are related to scattering data via a generalization of the well-known Levinson theorem [13]. A thorough discussion of the index, $\zeta$ - and $\eta$-functions in terms of boundary spectral functions for APS boundary problems can be found in $[14,15]$.

Alternatively, one may consider the boundary value problem for the Dirac operator acting on functions that satisfy local, bag-like, boundary conditions. These conditions are closely related to those appearing in the effective models of quark confinement known as MIT bag models [16], or their generalizations, the chiral bag models [17]. The physical motivation for studying these local boundary conditions is thus clear.

In this paper, we will study the Euclidean Dirac operator in two dimensions, acting on functions satisfying local bag boundary conditions $[18,19]$. Such boundary conditions are defined through the projector in equation (3). They contain a real parameter $\theta$, which is to be interpreted as an analytic continuation of the well-known $\theta$-parameter in gauge theories. Indeed, for $\theta \neq 0$, the effective actions for the Dirac fermions contain a $C P$-breaking term proportional to $\theta$ and proportional to the instanton number [19]; for example

$$
\begin{array}{ll}
\text { two dimensions } & \Gamma_{\text {eff }} \sim \theta \int \mathrm{d}^{2} x F_{01}+\cdots \\
\text { four dimensions } & \Gamma_{\text {eff }} \sim \theta \int \mathrm{d}^{4} x \epsilon_{\mu \nu \alpha \beta} F_{\mu \nu} F_{\alpha \beta}+\cdots .
\end{array}
$$

For $\theta \neq 0$ we will refer to the bag boundary conditions as chiral while, in the particular case $\theta=0$, we will call them non-chiral or pure MIT conditions. In both cases, the Dirac operator is self-adjoint. Moreover, in two dimensions, not only is the first-order boundary value problem elliptic, but so also is the associated second-order problem.

One of the main characteristics of bag boundary conditions is that they lead to an asymmetry in the non-zero spectrum. Thus, in this paper we will study the boundary contribution to the spectral asymmetry for bag boundary conditions in two-dimensional Euclidean space. The pure MIT case was studied, for any even dimension, in [20]. We will compare our results to those in this reference whenever adequate.

Note that, as in any even dimension, there is no volume contribution to the asymmetry (for a proof see, for instance, [4]; qualitatively, this is due to the existence of $\gamma_{5}$, which anticommutes with the Dirac operator). So, the boundary contribution is also the total asymmetry. In section 3, the asymmetry will be expressed in terms of spectral functions of the boundary operator $A$. Throughout our calculation in that section, we will assume the manifold to be of product type near the boundary and $A$ to be independent of the normal variable.

As an example of a product manifold we will evaluate, in section 4 , the asymmetry in a finite cylinder with twisted boundary conditions along the circle direction, imposing APS boundary conditions on one end of the cylinder and chiral bag conditions on the other end. The result will be shown to be consistent with our general prediction in section 3 .

In section 5, we will compute the spectral asymmetry in the case of a disc (two-dimensional bag), for chiral bag boundary conditions. Note this is a non-product case; however, we will suggest that the outcome of this calculation might be understood from our general result in section 3.

Finally, section 6 contains the generalization to the case in which certain gauge potentials are present, as well as some comments concerning the extension of our results to higher dimensions. 


\section{The heat kernel in terms of boundary eigenvalues}

In this section we rewrite the known heat kernel for the free Euclidean Dirac operator on the semi-infinite cylinder subject to bag boundary conditions, such that the spectral resolution with respect to the boundary operator becomes transparent. To this end, it is convenient to choose a chiral representation for the Euclidean $\gamma$-matrices in two dimensions,

$$
\gamma_{0}=\sigma_{1} \quad \gamma_{1}=\sigma_{2} \quad \text { and } \quad \gamma_{5}=-\mathrm{i} \gamma_{0} \gamma_{1}=\sigma_{3} .
$$

Then, the free Dirac operator takes the form

$$
P=\mathrm{i}\left(\gamma_{0} \partial_{0}+\gamma_{1} \partial_{1}\right)=\left(\begin{array}{cc}
0 & \partial_{1}+A \\
-\partial_{1}+A & 0
\end{array}\right)
$$

where $A$ is the boundary operator

$$
A=\mathrm{i} \partial_{0}
$$

which will play an important role in what follows. The Euclidean 'time' coordinate $0 \leqslant x_{0}<\beta$ is tangential to the boundary at $x_{1}=0$. The 'spatial' variable $x_{1} \geqslant 0$ is normal to the boundary and grows towards the interior of the semi-infinite cylinder. The projector defining the local bag boundary condition

$$
\left.B \psi\right|_{x_{1}=0}=0
$$

at the boundary $x_{1}=0$ reads

$$
B=\frac{1}{2}\left(1-\mathrm{i} \gamma_{5} \mathrm{e}^{\gamma_{5} \theta} \not h\right)=\frac{1}{2}\left(1+\mathrm{i} \gamma_{5} \mathrm{e}^{\gamma_{5} \theta} \gamma_{1}\right)=\frac{1}{2}\left(\begin{array}{cc}
1 & \mathrm{e}^{\theta} \\
\mathrm{e}^{-\theta} & 1
\end{array}\right)
$$

where $n^{\mu}$ is the outward-oriented normal, $n^{\mu}=(0,-1)$.

For convenience we introduce the variables $\xi_{\mu}=x_{\mu}-y_{\mu}$ and $\eta=x_{1}+y_{1}$. Then, the heat kernel of the associated second-order operator reads, in terms of the eigenvalues $a_{n}$ of the boundary operator $A$,

$$
\begin{aligned}
K(t, x, y)= & \frac{1}{\beta \sqrt{4 \pi t}} \sum_{n} \mathrm{e}^{\mathrm{i} a_{n} \xi_{0}} \mathrm{e}^{-a_{n}^{2} t}\left\{\mathrm{e}^{-\xi_{1}^{2} / 4 t} \mathbb{1}+\mathrm{e}^{-\eta^{2} / 4 t} M\right. \\
& \left.-N \tanh \theta \mathrm{e}^{-\eta^{2} / 4 t}\left[1-\frac{\sqrt{4 \pi t}}{\sinh 2 \theta} a_{n} \mathrm{e}^{u_{n}(\eta, t)^{2}} \operatorname{erfc}\left[u_{n}(\eta, t)\right]\right]\right\}
\end{aligned}
$$

where we introduced the abbreviation

$$
u_{n}(\eta, t)=\frac{\eta}{\sqrt{4 t}}-a_{n} \sqrt{t} \tanh \theta
$$

and the complementary error function,

$$
\operatorname{erfc}(x)=\frac{2}{\sqrt{\pi}} \int_{x}^{\infty} \mathrm{d} y \mathrm{e}^{-y^{2}} .
$$

Moreover, $\mathbb{1}$ denotes the $2 \times 2$ identity matrix,

$$
M=\left(\begin{array}{cc}
\mathrm{e}^{\theta} \sinh \theta & -\cosh \theta \\
-\cosh \theta & -\mathrm{e}^{-\theta} \sinh \theta
\end{array}\right) \quad \text { and } \quad N=\left(\begin{array}{cc}
\mathrm{e}^{\theta} & -1 \\
-1 & \mathrm{e}^{-\theta}
\end{array}\right) \sinh \theta
$$

For finite temperature field theory, in which case the Dirac field is anti-periodic in $x_{0}$ and hence the eigenvalues of the boundary operator are $a_{n}=2 \pi(n+1 / 2) / \beta$, the result (4) coincides with the Fourier transform of equation (101) in [21]. 


\section{Boundary contribution to the spectral asymmetry from bag boundary conditions}

As already commented, since the Euclidean spacetime is even dimensional, there is no bulk contribution to the asymmetry. To obtain the boundary contribution, the eigenvalue problem for the Dirac operator $P$ should be investigated on a collar neighbourhood of the boundary. Here, we consider instead the operator on the semi-infinite cylinder extending to $x_{1} \rightarrow \infty$. As is well known [20], since we are treating a self-adjoint problem, this yields the correct answer for an invertible boundary operator $A$. We shall discuss the non-invertible case towards the end of this section. Hence, for the moment, we assume $a_{n} \neq 0$ for all $n$.

Denoting the real eigenvalues of the Dirac operator by $\lambda$, the relevant spectral function is

$\eta(s, P)=\sum_{\lambda} \frac{\operatorname{sign} \lambda}{|\lambda|^{s}}=\zeta\left(\frac{s+1}{2}, P^{2}, P\right)=\frac{1}{\Gamma\left(\frac{s+1}{2}\right)} \int_{0}^{\infty} \mathrm{d} t t^{\frac{s-1}{2}} \operatorname{Tr}\left(P \mathrm{e}^{-t P^{2}}\right)$.

The Dirac trace can be computed with the help of

$$
\begin{array}{ll}
\operatorname{Tr}\left(\gamma_{0,1} \mathbb{1}\right)=\operatorname{Tr}\left(\gamma_{1} M\right)=\operatorname{Tr}\left(\gamma_{1} N\right)=0 \quad \text { and } \\
\operatorname{Tr}\left(\gamma_{0} M\right)=-2 \cosh \theta \quad \operatorname{Tr}\left(\gamma_{0} N\right)=-2 \sinh \theta .
\end{array}
$$

From (4) one obtains for the Dirac trace of the kernel needed in equation (5):

$$
\begin{aligned}
\operatorname{Tr}|x| P \mathrm{e}^{-t P^{2}}|y\rangle & =\frac{\cosh \theta \mathrm{e}^{-\eta^{2} / 4 t}}{\mathrm{i} \beta \sqrt{\pi t}} \operatorname{Tr}\left(\frac { \partial } { \partial x _ { 0 } } \sum _ { n } \mathrm { e } ^ { \mathrm { i } a _ { n } \xi _ { 0 } } \mathrm { e } ^ { - a _ { n } ^ { 2 } t } \left\{1-\tanh ^{2} \theta\right.\right. \\
& \left.\left.\times\left[1-\frac{a_{n} \sqrt{4 \pi t}}{\sin 2 \theta} \mathrm{e}^{u_{n}(\eta, t)^{2}} \operatorname{erfc}\left[u_{n}(\eta, t)\right]\right]\right\}\right)
\end{aligned}
$$

After performing the derivative with respect to $x_{0}$, setting $x_{\mu}=y_{\mu}$ and integrating over the tangential variable, one is left with the following integral over the normal variable $x_{1} \equiv x$ :

$\operatorname{Tr}\left(P \mathrm{e}^{-t P^{2}}\right)=\sum_{n} a_{n} \mathrm{e}^{-a_{n}^{2} t} \int_{0}^{\infty} \mathrm{d} x\left\{\frac{1}{\sqrt{\pi t}}+a_{n} \tanh \theta \mathrm{e}^{u_{n}^{2}(2 x, t)} \operatorname{erfc}\left[u_{n}(2 x, t)\right]\right\} \frac{\mathrm{e}^{-x^{2} / t}}{\cosh \theta}$

where we took into account that for $x_{\mu}=y_{\mu}$ we have

$$
u_{n}(\eta, t)=u_{n}(2 x, t)=\frac{x}{\sqrt{t}}-a_{n} \sqrt{t} \tanh \theta \quad x=x_{1} .
$$

Now, we may use the simple identity

$$
-\frac{1}{2} \frac{\partial}{\partial x}\left[\mathrm{e}^{-x^{2} / t+u_{n}^{2}(2 x, t)} \operatorname{erfc}\left[u_{n}(2 x, t)\right]\right]=\mathrm{e}^{-x^{2} / t}\left[\frac{1}{\sqrt{\pi t}}+a_{n} \tanh \theta \mathrm{e}^{u_{n}^{2}(2 x, t)} \operatorname{erfc}\left[u_{n}(2 x, t)\right]\right]
$$

to rewrite the relevant trace as follows:

$$
\begin{aligned}
\operatorname{Tr}\left(P \mathrm{e}^{-t P^{2}}\right) & =-\frac{1}{2 \cosh \theta} \sum_{n} a_{n} \mathrm{e}^{-a_{n}^{2} t / \cosh ^{2} \theta} \int_{0}^{\infty} \mathrm{d} x \frac{\partial}{\partial x}\left[\mathrm{e}^{-2 x a_{n} \tanh \theta} \operatorname{erfc}(u(2 x, t))\right] \\
& =\frac{1}{2} \sum_{n} \frac{a_{n}}{\cosh \theta} \mathrm{e}^{-a_{n}^{2} t / \cosh ^{2} \theta} \operatorname{erfc}\left[-\sqrt{t} \tanh \theta a_{n}\right] .
\end{aligned}
$$

The asymmetry is obtained by inserting (8) into (5) and, hence, it is given by $\eta(s, P)=\frac{1}{\Gamma\left(\frac{s+1}{2}\right)} \sum_{n} \frac{a_{n}}{2 \cosh \theta} \int_{0}^{\infty} \mathrm{d} t t^{\frac{s-1}{2}} \mathrm{e}^{-a_{n}^{2} t / \cosh ^{2} \theta}\left[1-\operatorname{erf}\left(-\sqrt{t} \tanh \theta a_{n}\right)\right]$

where erf is the error function,

$$
\operatorname{erf}(x)=1-\operatorname{erfc}(x)=\frac{2}{\sqrt{\pi}} \int_{0}^{x} \mathrm{~d} y \mathrm{e}^{-y^{2}} .
$$


Finally, changing variables to $\tau=a_{n}^{2} t / \cosh ^{2} \theta$, interchanging the order of the integrations and integrating over $\tau$ one obtains the following rather explicit expression:

$$
\begin{aligned}
\eta(s, P) & =\frac{1}{2} \cosh ^{s} \theta \sum_{n}\left(a_{n}^{2}\right)^{-s / 2}\left[\operatorname{sign}\left(a_{n}\right)+I(s, \theta)\right] \\
& =\frac{1}{2} \cosh ^{s} \theta\left[\eta(s, A)+\zeta\left(\frac{s}{2}, A^{2}\right) I(s, \theta)\right]
\end{aligned}
$$

where we have introduced the function

$$
I(s, \theta)=\frac{2}{\sqrt{\pi}} \frac{\Gamma\left(\frac{s}{2}+1\right)}{\Gamma\left(\frac{s}{2}+\frac{1}{2}\right)} \int_{0}^{\sinh \theta} \mathrm{d} x\left(1+x^{2}\right)^{-1-s / 2} .
$$

With $\pi I(0, \theta)=2 \arctan (\sinh \theta)$ we obtain

$$
\eta(0, P)=\frac{1}{2}\left\{\eta(0, A)+\frac{2}{\pi} \zeta\left(0, A^{2}\right) \arctan (\sinh \theta)\right\} .
$$

Now, the second term within the curly brackets can be seen to vanish, since the boundary is a closed manifold of odd dimensionality. In fact, in our case, $\zeta\left(0, A^{2}\right)=a_{1}\left(A^{2}\right)=0$, where $a_{1}\left(A^{2}\right)$ is a heat kernel coefficient in the notation of [4] (for details, see theorem 1.12.2 and lemma 1.8.2 in this reference), and we are left with

$$
\eta(0, P)=\frac{1}{2} \eta(0, A) \text {. }
$$

As far as $A$ is invertible, this is the main result of this section, relating the $\eta$-invariant of the Dirac operator to the same invariant of the boundary operator. Note that the outcome is the same irrespective of the value of $\theta$, i.e. it holds for both pure MIT and chiral bag conditions. The first case was treated before in [20]; our result coincides with the one given in that reference (equation (4.16)), up to an overall factor $1 / 2$. This discrepancy seems to be due to an extra factor of 2 in equations (4.7) and (4.8) in that reference. This extra factor is inconsistent with equation (4.10), and has seemingly propagated to theorem 4.4 in the same paper.

Our result (11) changes sign when the normal to the boundary points in the opposite direction, since then the non-diagonal entries in $M$ and $N$ change sign and, as a consequence, so does the Dirac trace.

As already pointed out, (11) gives the whole spectral asymmetry when the boundary Dirac operator $\gamma_{0} A$ is invertible. In fact, for such cases it was proved in [20] (see also [22]) that the asymmetry splits, in the adiabatic (infinite volume) limit, into the volume contribution plus the infinite cylinder one. Moreover, [23] shows that the spectral asymmetry is independent of the size of the manifold when the boundary value problem is self-adjoint, as in our case. This, together with the vanishing of the volume contribution in even dimensions, leads to the previous conclusion.

Now, we study the more subtle case of a non-invertible boundary operator $A$. Then, as can be seen from (7), $a_{n}=0$ would give no extra contribution in the semi-infinite cylinder. However, in this case, the trace (8) can differ in a substantial way from the corresponding one in the collar neighbourhood. As explained in [22], both large $t$ behaviours may be different, thus giving extra contributions to the asymmetry in the collar. This difference in high $t$ behaviour is due to the presence of 'small' eigenvalues, vanishing as the inverse of the size of the manifold in the adiabatic limit [24]. These extra contributions can be determined, modulo integers, by using the arguments in $[4,23,25]$. To this end, consider the one-parameter family of differential operators

$$
P_{\alpha}=P+\frac{2 \pi}{\beta} \alpha \gamma_{0} \quad P_{0}=P .
$$


These operators share the same $\alpha$-independent domain. They are invertible for $\alpha \neq 0$ and can be made invertible for all $\alpha$ by subtracting the projector on the subspace of small eigenvalues related to the zero modes at $\alpha=0$. This then yields a new family of operators $P_{\alpha}^{\prime}$ and one obtains

$$
\eta\left(0, P_{\alpha}\right)=\eta\left(0, P_{\alpha}^{\prime}\right) \bmod \mathbb{Z} \quad \text { and } \quad \frac{\mathrm{d}}{\mathrm{d} \alpha} \eta\left(0, P_{\alpha}\right)=\frac{\mathrm{d}}{\mathrm{d} \alpha} \eta\left(0, P_{\alpha}^{\prime}\right) .
$$

Then, differentiating with respect to $\alpha$ one finds

$$
\begin{aligned}
\frac{\mathrm{d}}{\mathrm{d} \alpha} \eta\left(0, P_{\alpha}^{\prime}\right) & =\left.\frac{1}{\Gamma\left(\frac{s+1}{2}\right)} \frac{\mathrm{d}}{\mathrm{d} \alpha} \int_{0}^{\infty} \mathrm{d} t t^{\frac{s-1}{2}} \operatorname{Tr}\left(P_{\alpha}^{\prime} \mathrm{e}^{-t P_{\alpha}^{\prime 2}}\right)\right|_{s=0} \\
& =\left.\frac{1}{\Gamma\left(\frac{s+1}{2}\right)} \int_{0}^{\infty} \mathrm{d} t t^{\frac{s-1}{2}} \operatorname{Tr}\left[\frac{\mathrm{d} P_{\alpha}^{\prime}}{\mathrm{d} \alpha}\left(1+2 t \frac{\mathrm{d}}{\mathrm{d} t}\right) \mathrm{e}^{-t P_{\alpha}^{\prime 2}}\right]\right|_{s=0} \\
& =-\frac{2 \pi s}{\beta \Gamma\left(\frac{s+1}{2}\right)} \int_{0}^{\infty} \mathrm{d} t t^{\frac{s-1}{2}} \operatorname{Tr}\left(\gamma_{0} \mathrm{e}^{-t P_{\alpha}^{\prime 2}}\right)+\left.\frac{4 \pi}{\beta \Gamma\left(\frac{s+1}{2}\right)} \operatorname{Tr}\left(t^{\frac{s+1}{2}} \gamma_{0} \mathrm{e}^{-t P_{\alpha}^{\prime 2}}\right)_{t=0}^{\infty}\right|_{s=0}
\end{aligned}
$$

where we performed a partial integration to arrive at the last equation. In addition, we used $\mathrm{d} P_{\alpha}^{\prime} / \mathrm{d} \alpha=\frac{2 \pi}{\beta} \gamma_{0}$. Since $P_{\alpha}^{\prime}-P_{\alpha}$ is an operator of finite range we may safely skip the prime in the last line of the above formula. Finally, the very last term in equation (12) can be seen to vanish, which gives, for the spectral flow (with almost the same calculation as the one starting with equation (6), except that no derivative w.r.t $x_{0}$ must be taken)

$\frac{\mathrm{d}}{\mathrm{d} \alpha} \eta\left(0, P_{\alpha}^{\prime}\right)=-\left.\frac{\pi}{\beta} \operatorname{Res}\right|_{s=0}\left[\zeta\left(\frac{s+1}{2}, A^{2}\right)+\frac{2}{\pi} \eta(s+1, A) \arctan (\sinh \theta)\right]$.

Now, the second term can be seen to vanish, since (again with the notation of [4]), $\left.\sqrt{\pi} \operatorname{Res}\right|_{s=0} \eta(s+1, A)=2 a_{0}\left(A^{2}, A\right)=0$. Moreover, $\left.\sqrt{\pi} \operatorname{Res}\right|_{s=0} \zeta\left(\frac{s+1}{2}, A^{2}\right)=2 a_{0}\left(A^{2}\right)=\frac{\beta}{\pi}$. Thus, one finally has for the spectral flow, irrespective of whether $A$ is invertible or not

$$
\frac{\mathrm{d}}{\mathrm{d} \alpha} \eta\left(0, P_{\alpha}\right)=-1 \text {. }
$$

So, at variance with the case treated in theorem 2.3 of [25], the spectral flow does not vanish for bag boundary conditions. As a consequence, the contribution to the asymmetry coming from boundary zero modes is different from an integer. This also seems to disagree with the result in [20]. Unfortunately, we were not able to trace the origin of this discrepancy from the results presented in that reference. However, we will see, in the next section, an explicit example of how this works.

\section{The asymmetry in a finite cylinder}

Here, we consider the simple case of the free Dirac operator on a finite 'cylinder' and impose twisted boundary conditions in the Euclidean time direction $\left(x^{0}\right.$ ranges from 0 to $\beta$ ), non-local APS boundary conditions at $x^{1}=0$ and local chiral bag boundary conditions at $x^{1}=L$. (Note that twisting the boundary fibre is equivalent to introducing a constant $A_{0}$ gauge field in the Dirac operator.)

The eigenfunctions of the Dirac operator (2) can be expanded in eigenfunctions of the boundary operator $A=\mathrm{i} \partial_{0}$, satisfying twisted boundary conditions in the time-direction with twist parameter $\alpha, \psi\left(x_{0}+\alpha\right)=\mathrm{e}^{2 \pi \mathrm{i} \alpha} \psi\left(x_{0}\right)$, as follows:

$$
\psi=\sum_{n} \psi_{n}\left(x_{1}\right) \mathrm{e}^{\mathrm{i} a_{n} x_{0}} \quad \psi_{n}=\left(\begin{array}{c}
f_{n} \\
g_{n}
\end{array}\right)
$$




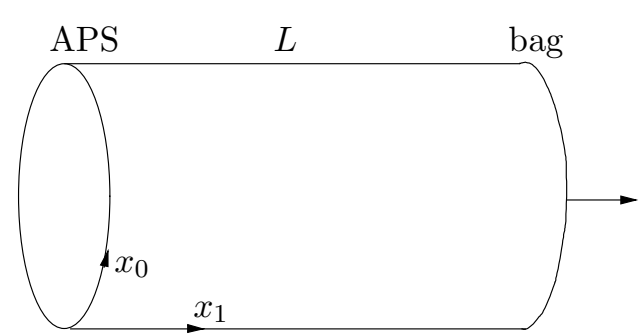

Figure 1. The scenario in this section.

where the eigenvalues of the boundary operator read

$$
a_{n}=\frac{2 \pi}{\beta}(n+\alpha) \quad n \in \mathbb{Z}
$$

For definiteness, we will consider $0 \leqslant \alpha<1$ such that $a_{n} \geqslant 0$ is equivalent to $n \geqslant 0$ and $a_{n}<0$ to $n<0$. A vanishing $\alpha$ corresponds to periodic boundary conditions, and $\alpha=1 / 2$ to anti-periodic (finite temperature) boundary conditions. The mode-functions in (15) fulfil the simple differential equations

$$
g_{n}^{\prime}-a_{n} g_{n}=\lambda f_{n} \quad \text { and } \quad-f_{n}^{\prime}-a_{n} f_{n}=\lambda g_{n} .
$$

At $x^{1}=0$, the APS boundary conditions require

$$
a_{n} \geqslant 0 \quad f_{n}(0)=0 \quad \text { and } \quad a_{n}<0 \quad g_{n}(0)=0 .
$$

Hence, the mode-functions have the form

$\psi_{n \geqslant 0} \sim\left(\begin{array}{c}\lambda \sinh \mu x_{1} \\ -a_{n} \sinh \mu x_{1}-\mu \cosh \mu x_{1}\end{array}\right) \quad \psi_{n<0} \sim\left(\begin{array}{c}-a_{n} \sinh \mu x_{1}+\mu \cosh \mu x_{1} \\ \lambda \sinh \mu x_{1}\end{array}\right)$

with $\mu=\sqrt{a_{n}^{2}-\lambda^{2}}$. On these we must impose chiral bag boundary conditions at $x_{1}=L$. The projector defining these conditions reads

$$
B=\frac{1}{2}\left(1-\mathrm{i} \gamma_{5} \mathrm{e}^{\gamma_{5} \theta} \not h\right)=\frac{1}{2}\left(\begin{array}{cc}
1 & -\mathrm{e}^{\theta} \\
-\mathrm{e}^{-\theta} & 1
\end{array}\right)
$$

and yields the following transcendental equations:

$$
\begin{array}{ll}
\left(\lambda \mathrm{e}^{-\theta}+a_{n}\right) \sinh L \mu_{n}(\lambda)+\mu_{n}(\lambda) \cosh L \mu_{n}(\lambda)=0 & \text { for } \quad n \geqslant 0 \\
\left(\lambda \mathrm{e}^{\theta}+a_{n}\right) \sinh L \mu_{n}(\lambda)-\mu_{n}(\lambda) \cosh L \mu_{n}(\lambda)=0 & \text { for } n<0
\end{array}
$$

for the eigenvalues $\lambda(\alpha)$ of the Dirac operator on the finite cylinder with APS and bag boundary conditions. With the evident relation

$$
a_{-n-1}(\alpha)=-a_{n}(1-\alpha)
$$

one shows that the assignment

$$
(n, \alpha, \theta, \lambda) \longrightarrow(-n-1,1-\alpha,-\theta,-\lambda)
$$

maps one of the lines of equation (16) into the other. Hence, it suffices to consider the case $n \geqslant 0$. The contribution of the negative $n$ to spectral functions is taken into account by exploiting the symmetry (17).

Let us first study the asymmetry for $\alpha \neq 0$, thus excluding the case of a non-invertible $A$. From the well-known formula

$$
\sum_{\lambda} \lambda^{-s}=\frac{1}{2 \pi \mathrm{i}} \oint_{\Gamma} \frac{\mathrm{d} z}{z^{s}} \frac{f^{\prime}(z)}{f(z)}
$$


with $f$ defined by the left-hand side in the first line of equation (16), one obtains

$$
\begin{gathered}
\eta(s, P)=\frac{1}{2 \pi \mathrm{i}} \sum_{n=0}^{\infty} \int_{\Gamma} \frac{\mathrm{d} z}{z^{s}} \frac{\mathrm{d}}{\mathrm{d} z} \log \frac{\left(a_{n}+z \mathrm{e}^{-\theta}\right) \sinh L \mu_{n}(z)+\mu_{n}(z) \cosh L \mu_{n}(z)}{\left(a_{n}-z \mathrm{e}^{-\theta}\right) \sinh L \mu_{n}(z)+\mu_{n}(z) \cosh L \mu_{n}(z)} \\
-(\alpha \rightarrow 1-\alpha, \theta \rightarrow-\theta)
\end{gathered}
$$

with $\mu_{n}(z)=\sqrt{a_{n}-z^{2}}$. The curve $\Gamma$ comes from $\infty+\mathrm{i} \epsilon$ to a small semi-circle avoiding the origin and goes back to $+\infty-\mathrm{i} \epsilon$, surrounding the real positive axis anti-clockwise.

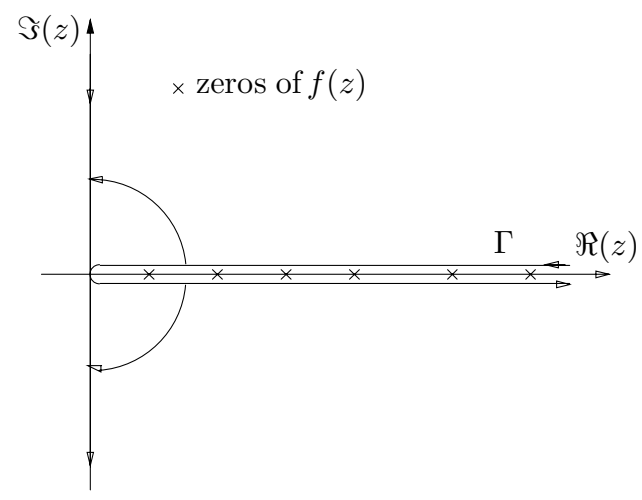

Figure 2. The integration curve.

Now, the contour can be opened to the imaginary axis, and the circle around the origin can be shrunk, since the integrand vanishes at $z=0$. After doing so, one gets

$$
\begin{gathered}
\eta(s, P)=\frac{1}{\mathrm{i} \pi} \sum_{n=0}^{\infty} \int_{0}^{\infty} \frac{\mathrm{d} t}{t^{s}} \cos \left(\frac{\pi s}{2}\right) \frac{\mathrm{d}}{\mathrm{d} t} \log \frac{\left(a_{n}-\mathrm{i} t \mathrm{e}^{-\theta}\right) \tanh L \mu_{n}(\mathrm{i} t)+\mu_{n}(\mathrm{i} t)}{\left(a_{n}+\mathrm{i} t \mathrm{e}^{-\theta}\right) \tanh L \mu_{n}(\mathrm{i} t)+\mu_{n}(\mathrm{i} t)} \\
-(\alpha \rightarrow 1-\alpha, \theta \rightarrow-\theta) .
\end{gathered}
$$

Now, changing variables according to $t=a_{n} u$, one obtains

$$
\begin{gathered}
\eta(s, P)=\frac{1}{\mathrm{i} \pi} \sum_{n=0}^{\infty} \cos \left(\frac{\pi s}{2}\right) a_{n}^{-s} \int_{0}^{\infty} \frac{\mathrm{d} u}{u^{s}} \frac{\mathrm{d}}{\mathrm{d} u} \log \frac{\left(1-\mathrm{i} u \mathrm{e}^{-\theta}\right) \tanh \left[L a_{n} \sqrt{1+u^{2}}\right]+\sqrt{1+u^{2}}}{\left(1+\mathrm{i} u \mathrm{e}^{-\theta}\right) \tanh \left[L a_{n} \sqrt{1+u^{2}}\right]+\sqrt{1+u^{2}}} \\
-(\alpha \rightarrow 1-\alpha, \theta \rightarrow-\theta) .
\end{gathered}
$$

The whole expression can be evaluated at $s=0$, and one obtains the following simple result for the spectral asymmetry

$$
\eta(0, P)=-\frac{1}{2}\left[\zeta_{H}(0, \alpha)-\zeta_{H}(0,1-\alpha)\right]=\alpha-\frac{1}{2}
$$

where $\zeta_{H}$ is the Hurwitz $\zeta$-function. In particular, the asymmetry vanishes in the finite temperature case $\left(\alpha=\frac{1}{2}\right)$.

As shown in the previous section (equation (11)), bag boundary conditions give, in the absence of boundary zero modes, a contribution $-\frac{1}{2} \eta(0, A)$ to the asymmetry. The minus sign is due to the fact that, at $x_{1}=L$, the external normal is $(0,1)$. APS boundary conditions give no contribution at all and, as a consequence, the total asymmetry is due to bag boundary conditions. In this case, it can be easily computed in terms of Hurwitz zeta functions

$$
\left.-\frac{1}{2} \eta(0, A)=-\frac{1}{2}\left(\frac{2 \pi}{\beta}\right)^{-s}\left[\sum_{n \geqslant 0}(n+\alpha)^{-s}-\sum_{n>0}(n-\alpha)^{-s}\right)\right]\left.\right|_{s=0}
$$

which reduce to equation (20). 
Let us finally study the periodic case, where a boundary zero mode does exist. The total asymmetry can be obtained as follows. From the symmetry (17) it follows, that

$$
(n, \theta, \lambda) \longrightarrow(-n,-\theta,-\lambda) \quad n \neq 0
$$

is a symmetry of equations (16). The contribution from these modes can be evaluated as in the invertible case and is found to be $\frac{1}{2}-\frac{2}{\pi} \arctan \mathrm{e}^{\theta}$. Regarding $n=0$, the contribution coming from these modes can be computed directly in terms of Hurwitz zeta functions, and it gives $-1+\frac{2}{\pi} \arctan \mathrm{e}^{\theta}$. So, the sum of both contributions gives for the total asymmetry $\eta(0, P)=-\frac{1}{2}$.

This result is again in complete agreement with our general result in the previous section. In fact, APS boundary conditions do not contribute to the asymmetry $\bmod \mathbb{Z}$. The contribution of the local boundary conditions $\bmod \mathbb{Z}$ can be obtained from the spectral flow in equation (12). Hence,

$$
\eta\left(0, P_{0}\right)-\eta\left(0, P_{1 / 2}\right)=\eta\left(0, P_{0}\right)=-\frac{1}{2}(\bmod \mathbb{Z}) .
$$

It is interesting to note that in all cases, bag boundary conditions transform the would-be contribution to the index due to APS boundary conditions into a spectral asymmetry. In fact, the problem can be easily seen to present no zero modes.

\section{Spectral asymmetry in the disc}

In this section, we will study the spectral asymmetry for the free Dirac operator in a disc, subject to bag boundary conditions at radius $R$ and with arbitrary $\theta$. Note that we are dealing with a non-product case. However, we will suggest a plausible interpretation in terms of our results in section 3. The Dirac operator on the disc, subject to non-local APS conditions, has been carefully analysed in $[7,13]$. In particular, the connection to the scattering theory of $P^{2}$ has been clarified in [13].

We choose the same chiral representation as in section 2 and take polar coordinates $(r, \varphi)$, such that the free Dirac operator takes the form

$P=\mathrm{i}\left(\gamma_{r} \partial_{r}+\gamma_{\varphi} \frac{\partial_{\varphi}}{r}\right) \quad$ with $\quad \gamma_{r}=\left(\begin{array}{cc}0 & \mathrm{e}^{-\mathrm{i} \varphi} \\ \mathrm{e}^{\mathrm{i} \varphi} & 0\end{array}\right) \quad \gamma_{\varphi}=\left(\begin{array}{cc}0 & -\mathrm{ie}^{-\mathrm{i} \varphi} \\ \mathrm{i} \mathrm{e}^{\mathrm{i} \varphi} & 0\end{array}\right)$.

Here, the angle $\varphi$ is the boundary variable, and $0 \leqslant r \leqslant R$ is the outward-growing normal one. With $h=\gamma_{r}$ the projector defining bag boundary conditions at $r=R$ reads

$$
B=\frac{1}{2}\left(1-\mathrm{i} \gamma_{5} \mathrm{e}^{\gamma_{5} \theta} \gamma_{r}\right)=\frac{1}{2}\left(\begin{array}{cc}
1 & -\mathrm{ie} \mathrm{e}^{\theta-\mathrm{i} \varphi} \\
\mathrm{ie} & 1
\end{array}\right)
$$

and the boundary operator at $r=R$ is

$$
A=\frac{\mathrm{i}}{R} \partial_{\varphi}
$$

We expand the eigenfunctions of the Dirac operator $P$ in eigenfunctions of the total angular momentum operator

$$
J=\frac{1}{\mathrm{i}} \frac{\partial}{\partial \varphi}+\frac{1}{2} \sigma_{3}
$$

which commutes with both $P$ and $B$,

$$
\psi=\sum_{n=-\infty}^{\infty} c_{n}\left(\begin{array}{c}
f_{n}(r) \mathrm{e}^{\mathrm{i} n \varphi} \\
g_{n}(r) \mathrm{e}^{\mathrm{i}(n+1) \varphi}
\end{array}\right)
$$


The radial mode-functions are determined by the differential equation $P \psi=\lambda \psi$, together with the bag boundary conditions. The differential equation implies

$$
f_{n}=J_{n}(|\lambda| r) \quad \text { and } \quad g_{n}=-\mathrm{i} \operatorname{sign}(\lambda) J_{n+1}(|\lambda| r)
$$

where $J_{n}$ is the Bessel function of integer order $n$. The boundary conditions with boundary operator (22) yield

$$
J_{n}(|\lambda| R)-\mathrm{e}^{\theta} \operatorname{sign}(\lambda) J_{n+1}(|\lambda| R)=0 \quad n \in \mathbb{Z} .
$$

Here it is convenient to consider these conditions for positive and negative eigenvalues $\lambda$ separately. With the help of $J_{-n}(x)=(-)^{n} J_{n}(x)$ they can be written as follows:

$$
\begin{array}{ll}
\lambda>0 & J_{n}(|\lambda| R)-\mathrm{e}^{\theta} J_{n+1}(|\lambda| R)=J_{n}(|\lambda| R)+\mathrm{e}^{-\theta} J_{n+1}(|\lambda| R)=0 \\
\lambda<0 & J_{n}(|\lambda| R)+\mathrm{e}^{\theta} J_{n+1}(|\lambda| R)=J_{n}(|\lambda| R)-\mathrm{e}^{-\theta} J_{n+1}(|\lambda| R)=0
\end{array}
$$

where $n=0,1,2, \ldots$. Note that these conditions are left invariant by the replacement

$$
(\lambda, \theta) \longrightarrow(-\lambda,-\theta) .
$$

Hence, with the help of (18) the spectral asymmetry is given by the following contour integral in the complex plane:

$$
\eta(s, P)=\frac{1}{2 \pi \mathrm{i}} \sum_{n=0}^{\infty} \int_{\Gamma} \mathrm{d} z z^{-s} \frac{\mathrm{d}}{\mathrm{d} z} \log \left(\frac{J_{n}(z R)-\mathrm{e}^{\theta} J_{n+1}(z R)}{J_{n}(z R)+\mathrm{e}^{\theta} J_{n+1}(z R)}\right)-(\theta \rightarrow-\theta)
$$

where the contour $\Gamma$ is the same as that in (19). Again we deform the path of integration such that we integrate along the imaginary axis. After doing that, and using the definition of the modified Bessel functions,

$$
J_{n}(\mathrm{i} x)=\mathrm{i}^{n} I_{n}(x) \quad \text { and } \quad J_{n}(-\mathrm{i} x)=(-\mathrm{i})^{n} I_{n}(x)
$$

we obtain

$$
\eta(s, P)=\frac{1}{\mathrm{i} \pi} \cos \frac{\pi s}{2} \sum_{n=0}^{\infty} \int_{0}^{\infty} \mathrm{d} t t^{-s} \frac{\mathrm{d}}{\mathrm{d} t} \log \frac{I_{n}(t R)+\mathrm{ie}^{\theta} I_{n+1}(t R)}{I_{n}(t R)-\mathrm{ie}^{\theta} I_{n+1}(t R)}-(\theta \rightarrow-\theta) .
$$

It is convenient to separate the contribution from $n=0$, which can be evaluated at $s=0$ without problems. The corresponding integral gives

$$
\begin{aligned}
\eta^{n=0}(0, P) & =\left.\frac{1}{\mathrm{i} \pi} \log \frac{I_{0}(t R)+\mathrm{ie}^{\theta} I_{1}(t R)}{I_{0}(t R)-\mathrm{ie}^{\theta} I_{1}(t R)}\right|_{0} ^{\infty}-(\theta \rightarrow-\theta) \\
& =\frac{1}{\mathrm{i} \pi} \log \frac{1+\mathrm{ie}^{\theta}}{1-\mathrm{ie}^{\theta}}-(\theta \rightarrow-\theta)=\frac{4}{\pi}\left[\frac{\pi}{4}-\arctan \mathrm{e}^{-\theta}\right] .
\end{aligned}
$$

For the remaining subspaces, $n \neq 0$, we add and subtract the first term in the Debye expansion of the modified Bessel functions.

To this end, we change variables according to $t=n u / R$, so that

$$
\eta^{n \neq 0}(s, P)=\frac{1}{\mathrm{i} \pi} \cos \frac{\pi s}{2} \sum_{n=1}^{\infty}\left(\frac{n}{R}\right)^{-s} \int_{0}^{\infty} \mathrm{d} u u^{-s} \frac{\mathrm{d}}{\mathrm{d} u} \log \frac{I_{n}(n u)+\mathrm{ie}^{\theta} I_{n+1}(n u)}{I_{n}(n u)-\mathrm{ie}^{\theta} I_{n+1}(n u)}-(\theta \rightarrow-\theta) .
$$

The first term in the Debye expansion of the argument of the logarithm gives

$$
\log \frac{I_{n}(n u)+\mathrm{ie}^{\theta} I_{n+1}(n u)}{I_{n}(n u)-\mathrm{ie}^{\theta} I_{n+1}(n u)} \sim \log F(u, \theta) \quad F(u, \theta)=-\frac{\sqrt{1+u^{2}}-1-\mathrm{i} u \mathrm{e}^{-\theta}}{\sqrt{1+u^{2}}-1+\mathrm{i} u \mathrm{e}^{-\theta}} .
$$


When this is added and subtracted in equation (27), the subtracted part can be seen to vanish at $s=0$, since the integrand cancels at both 0 and $\infty$. Thus, we are left with

$$
\eta^{n \neq 0}(s, P)=\frac{1}{\mathrm{i} \pi} \cos \frac{\pi s}{2} \sum_{n=1}^{\infty}\left(\frac{n}{R}\right)^{-s} \int_{0}^{\infty} \mathrm{d} u u^{-s} \frac{\mathrm{d}}{\mathrm{d} u} \log F(u, \theta)-(\theta \rightarrow-\theta)
$$

which yields a finite expression for $s=0$,

$$
\eta^{n \neq 0}(0, P)=\left.\frac{1}{\mathrm{i} \pi} \zeta_{R}(0) \log F(u, \theta)\right|_{0} ^{\infty}-(\theta \rightarrow-\theta)
$$

where $\zeta_{R}$ is the Riemann zeta function. Inserting the values of $F$ and $\zeta_{R}(0)$ yields

$$
\eta^{n \neq 0}(0, P)=\frac{2}{\pi}\left[\arctan \mathrm{e}^{-\theta}-\frac{\pi}{4}\right]
$$

When added to the contribution in equation (26), this gives for the total asymmetry

$$
\eta(0, P)=\frac{2}{\pi}\left[\arctan \mathrm{e}^{|\theta|}-\frac{\pi}{4}\right] .
$$

This is precisely the result predicted by equation (10) when the eigenvalues of $A$ are of the form $a_{n}=n / R$, with $n \in \mathbb{Z}$. In fact, in this case, $\eta(0, A)=0$ and $\zeta^{\prime}\left(0, A^{2}\right)=-1$ (the latter is evaluated in the subspace orthogonal to the zero mode). This can be interpreted as follows: The operator $P$ in equation (21) is not of the form (2). However, it reduces to such a form (although with an $r$-dependent $A$ ) after choosing the eigenfunctions as in (23). Now, due to the different dependence on the tangent variable of both components in the spinors, $\gamma_{0} A$ never goes through zero modes and the calculation in the infinite cylinder seemingly gives the correct value for the asymmetry, even though this is a non-product example.

\section{Comments}

As already pointed out, our result in equation (11) gives the answer also in the presence of a constant $A_{0}$ gauge field, which can always be eliminated with the only consequence of twisting the boundary fibre. It can also be shown to hold for a gauge field such that $A_{0}=A_{0}\left(x_{0}\right)$ (independent of the normal variable) and $A_{1}=0$ for, then, a gauge transformation can be performed of the form $\psi^{\prime}\left(x_{0}, x_{1}\right)=\mathrm{e}^{\mathrm{i} \int_{0}^{x_{0}} A_{0}(x) \mathrm{d} x} \psi\left(x_{0}, x_{1}\right)$, again leading to just a twist in the boundary fibre.

Finally, our result should, in principle, extend to higher even dimensions. It is clear that this is so in the case of pure MIT bag boundary conditions $(\theta=0)$. For chiral bag boundary conditions, this is not so clear due to the presence of oblique boundary conditions [26] (and possible lack of ellipticity) in the associated second-order problem [1, 27, 28].

\section{Acknowledgments}

We thank H Falomir and D V Vassilevich for carefully reading the manuscript and for useful suggestions. This work was partially supported by Fundación Antorchas and DAAD (grant 13887/1-87), CONICET (grant 0459/98) and UNLP (grant X298).

\section{References}

[1] Kirsten K 2001 Spectral Functions in Mathematics and Physics (London/Boca Raton, FL: Chapman and Hall/CRC Press)

[2] Dowker J S and Critchley R 1976 Phys. Rev. D 133224

Hawking S W 1977 Commun. Math. Phys. 55133 
[3] Elizalde E, Odintsov S D, Romeo A, Bytsenko A A and Zerbini S 1994 Zeta Regularization Techniques with Applications (Singapore: World Scientific)

Bordag M, Mohiden U and Mostepanenko V M 2001 Preprint quant-ph/0106045

Santangelo E M 2002 Theor. Math. Phys. 131607

[4] Gilkey P B 1995 Invariance Theory, the Heat Equation, and the Atiyah-Singer Index Theorem (Boca Raton, FL: CRC Press)

[5] Atiyah M F, Patodi V K and Singer I M 1975 Math. Proc. Camb. Phil. Soc. 7743

Atiyah M F, Patodi V K and Singer I M 1976 Math. Proc. Camb. Phil. Soc. 78495

Atiyah M F, Patodi V K and Singer I M 1980 Math. Proc. Camb. Phil. Soc. 7971

See also Eguchi T, Gilkey R and Hanson A 1980 Phys. Rep. 66213

[6] Roemer H and Schroer P B 1977 Phys. Lett. B 21182

[7] Ninomiya M and Tan C I 1985 Nucl. Phys. B 245199

Ma Z Q 1986 J. Phys. A: Math. Gen. 19 L317

[8] D'Eath P D and Esposito G 1991 Phys. Rev. D 441713

[9] Beneventano C G, De Francia M and Santangelo E M 1999 Int. J. Phys. A 144749

[10] Vassilevich D V 2001 J. High Energy Phys. JHEP03(2001)023

[11] Niemi A J and Semenoff G W 1984 Phys. Rev. D 30809

[12] Elizalde E, Cognola G and Zerbini S 1999 Dirac functional determinant in terms of the $\eta$ invariant and the noncommutative residue Preprint hep-th/9910038

[13] Musto R, O’Raifeartaigh L and Wipf A 1998 Phys. Lett. B 175433

Forgacs P, O'Raifeartaigh L and Wipf A 1987 Nucl. Phys. B 293559

[14] Grubb G and Seeley R T 1996 J. Geom. Anal. 631

[15] Wojciechowski K P 1991 Commun. Math. Phys. 142139

[16] Chodos A, Jaffe R L, Johnson K and Thorn C B 1974 Phys. Rev. D 93471

[17] Rho M, Goldhaber A S and Brown G E 1983 Phys. Rev. Lett. 51747

[18] Hraskó P and Balog J 1984 Nucl. Phys. B 245118

[19] Durr S and Wipf A 1995 Nucl. Phys. B 443201

[20] Klimek S and Wojciechowski K P 1996 J. Funct. Anal. 136269

[21] Durr S and Wipf A 1997 Ann. Phys., NY 255333

[22] Douglas R G and Wojciechowski K P 1991 Commun. Math. Phys. 142139

[23] Müller W 1994 J. Differ. Geom. 40311

[24] Park J and Wojciechowski K 2001 Preprint math.DG/0111046

[25] Wojciechowski K P 1995 Commun. Math. Phys. 169315

[26] Kirsten K 2001 Private communication

[27] Dowker J S and Kirsten K 1997 Class. Quantum Grav. 14 L169

[28] Avramidi I G and Esposito G 1998 Class. Quantum Grav. 151141 